\title{
Impact of chrysosplenetin on the
} pharmacokinetics and anti-malarial efficacy of artemisinin against Plasmodium berghei as well as in vitro CYP450 enzymatic activities in rat liver microsome

Shijie Wei ${ }^{1,2+}$, Hongyan $\mathrm{Ji}^{2+}$, Bei Yang ${ }^{1}$, Liping Ma ${ }^{1}$, Zhuchun Bei ${ }^{3}$, Xiang $\mathrm{Li}^{1}$, Hongwan Dang ${ }^{2}$, Xiaoying Yang ${ }^{2}$, Cheng Liu', Xiuli Wu' and Jing Chen ${ }^{1 *}$

\begin{abstract}
Background: Artemisinin (ART) is an efficacious and safe anti-malarial drugs but has low oral bioavailability and auto-induction profiles during multiple dosing. The pharmacokinetic disadvantages have been found to partially depend on the induction of cytochrome P-450 enzymes by ART and resulted in the therapeutic failure due to insufficient drug levels. The present study, therefore, investigated the impacts of chrysosplenetin (CHR), a polymethoxylated flavonoid from Artemisia annua, on the pharmacokinetics and the anti-malarial efficacy of ART against Plasmodium berghei. The inhibition of CHR on enzymatic activity of CYP1A2, CYP2A, CYP2C19, CYP2D6, CYP2E1, and CYP3A in rat liver microsome was also investigated. IC50, Km, Ki, and inhibitory type of CHR were respectively calculated.

Methods: Twenty rats were randomly divided into four groups and received three-day oral doses of ART in absence or presence of CHR (in ratio of 1:0, 1:1, 1:2, and 1:4, respectively). Plasma samples were separately harvested for ART pharmacokinetics analysis using a valid liquid chromatography tandem mass spectrometric (LC-MS/MS) method. Female Kunming mice were inoculated by P. berghei K173 strain and pre-exposed to three-day oral administration of ART with or without CHR as pharmacokinetics protocol. Giemsa staining method was applied to calculate percent parasitaemia (\%) and inhibition (\%). In vitro rat liver microsomal model was employed to elucidate the inhibitory effect of CHR on CYP1A2, CYP2A, CYP2C19, CYP2D6, CYP2E1, and CYP3A.

Results: The $A U C_{0-t^{\prime}} C_{\text {max }}$ and $t_{1 / 2}$ of ART increased significantly $(P<0.05$ or $P<0.01)$ as well as declined $C L z(P<0.05$ or $P<0.01)$ after three-day oral doses of ART in presence of CHR (1:2) when compared with ART alone. Also, parasitaemia (\%) remarkably attenuated 1.59 folds with 1.63-fold augmented inhibition (\%) when the ratio between ART and CHR reached 1:2. CHR itself had no anti-malarial efficacy $(P>0.05)$. CHR inhibited in vitro activity of CYP1A2 and CYP2C19 $\left(P<0.01, I C_{50}=4.61\right.$ and $\left.6.23 \mu \mathrm{M}\right)$ in a concentration-response manner. The inhibition did not emerge on CYP2E1 and CYP3A until the CHR concentration exceeded $4.0 \mu \mathrm{M}\left(P<0.01, I C_{50}=28.17\right.$ and $\left.3.38 \mu \mathrm{M}\right)$. CHR has no impact on CYP 2A and CYP2D6 ( $P>0.05)$. The inhibition types of CHR on CYP1A2 and CYP3A belonged to noncompetitive and uncompetitive, respectively.
\end{abstract}

\footnotetext{
*Correspondence: chenjing2005@126.com

†Shijie Wei and Hongyan Ji contributed equally to the manuscript

${ }^{1}$ School of Pharmacy, Ningxia Medical University, 1160\# Shengli Street,

Xingqing District, Yinchuan 750004, People's Republic of China

Full list of author information is available at the end of the article
} 
Conclusions: Co-administration of ART with CHR in ratio of 1:2 achieved a synergic anti-malarial effect partly because of the noncompetitive or uncompetitive inhibition of CHR of drug-metabolism enzymes, especially CYP3A which is closely related to the auto-induction of ART.

Keywords: Chrysosplenetin, Artemisinin, Pharmacokinetics, Anti-malarial efficacy, Rat liver microsome, CYP3A

\section{Background}

Artemisinins (ART) (Fig. 1a) anti-malarial drugs belong to the sesquiterpene lactone family containing the specific endoperoxide bridge. They have been successfully used for more than two decades in the clinical treatment of malaria in regions with multi-drug resistant Plasmodium falciparum [1]. Up to now, ART and its semisynthetic derivatives are still the most important anti-malarial drugs available and ART-based combination therapy (ACT) has been recommended worldwide as first-line treatment for falciparum malaria since 2006 [2, 3]. Despite its widespread use, ART has very unusual pharmacokinetic properties with saturable first-pass hepatic metabolism and time-dependent pharmacokinetics during repeated oral administration [4-6].
ART, therefore, has very low oral bioavailability, merely $8-10 \%$. The auto-induction of both phase I and phase II metabolism of ART was demonstrated to be present in healthy Chinese subjects after a recommended two-day oral dose of ART-piperaquine probably due to the induction of CYP2B6 and CYP3A4 enzyme activity [7]. It was reported that ART in vitro metabolism was mediated primarily by CYP2B6, with a minor contribution from CYP2A6 and CYP3A4 [8]. El-Lakkany et al. [9] found that coadministration of grapefruit juice with artemether $(150 \mathrm{mg} / \mathrm{kg})$ eliminated eggs and granulomatous reactions and achieved complete protection of the host from damage induced by schistosomal infection because of the inhibitory effects of grapefruit juice on CYP450 and cyt b5.

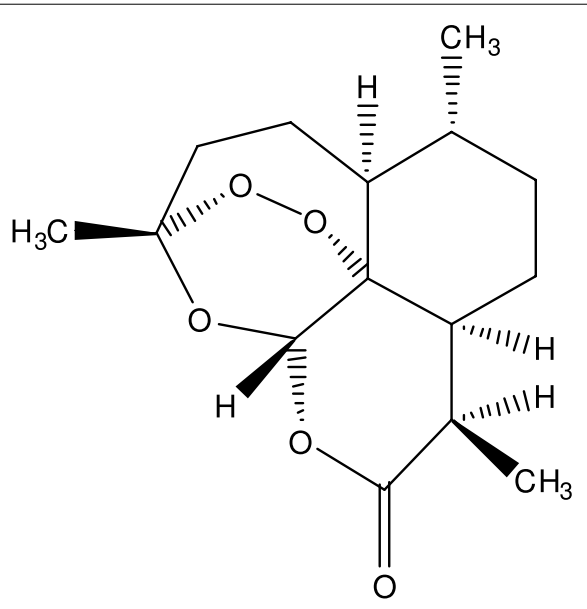

a

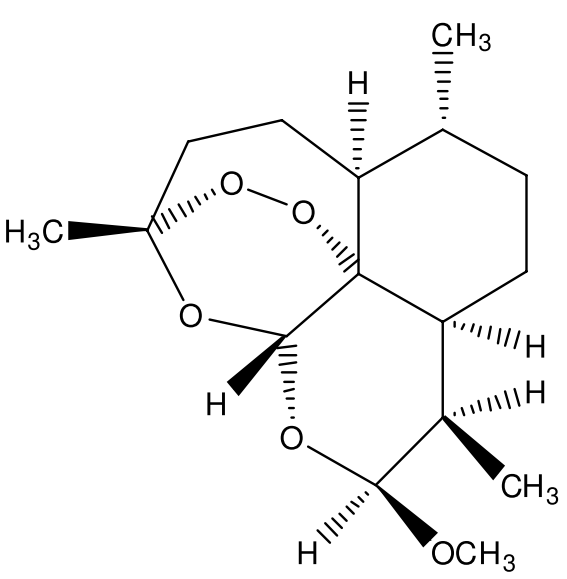

b<smiles>COc1cc(-c2oc3cc(OC)c(OC)c(O)c3c(=O)c2OC)ccc1O</smiles>

Fig. 1 Structure of ART (a), ARM (b), and CHR (c) 
Literature has shown that several polymethoxyflavonoid constituents from Artemisia annua, such as artemetin, casticin, chrysosplenetin (CHR, Fig. 1c) and chrysosplenol D may contribute to the activity of ART against $P$. falciparum $[10,11]$. However, the mechanism of action has not yet been well defined. Generally, most flavonoids have an inhibitory effect on CYP450 enzymes and the aglycones have a stronger inhibition than glycosides [12, 13]. CHR has been previously enriched from the industrial wastes of ART about $1 \mathrm{~g}$ (over $98 \%$ purity) and a China National Invention Patent (ZL201210093926.0, China) has been awarded. Structure of CHR was identified by ${ }^{1} \mathrm{H}-\mathrm{NMR},{ }^{13} \mathrm{C}-\mathrm{NMR}$ and $2 \mathrm{D}-\mathrm{NMR}$ [14-19]. The present study was designed to investigate the impact of $\mathrm{CHR}$ on the pharmacokinetics and the anti-malarial efficacy of ART against Plasmodium berghei. Further, the inhibition of CHR on in vitro CYP450 enzymatic activity was also elucidated. It was demonstrated here that CHR increased the AUC, $\mathrm{C}_{\max }$, and $t_{1 / 2}$ of ART along with a decreased CLz and enhanced its in vivo anti-malarial efficacy against malarial parasites infection, partially due to the uncompetitive inhibition on CYP3A. The data presented in this paper indicate the promising tendency for CHR to be used as CYP 450 enzymatic inhibitor in coordination with ART-type anti-malarial drugs.

\section{Methods}

\section{Chemicals and reagents}

Artemisinin and internal standard artemether (ARM, Fig. 1b) were purchased from Chongqing Huali Konggu Co., Ltd. (purity >99.0 \%, Chongqing, China). CHR (purity >98.0\%) was previously isolated and purified from the industrial wastes of ART (acetone layer) with about $1 \%$ yield in the authors' laboratory. Industrial waste was kindly gifted by Chongqing Huali Konggu Co. Ltd, and the voucher specimen of the waste has been deposited in the College of Pharmacy, Ningxia Medical University, for further references.

Six metabolic probes including phenacetin $(\mathrm{PN})$, coumarin (CA), omeprazole (OMP), dextromethophan (DM), and chlorzoxazone (CLZ) were purchased from National Institutes for Food and Drug Control, China. Midazolam (MDZ) injection was provided by Jiangsu Enhua Pharmaceutical Co., Ltd $(10 \mathrm{mg} / 2 \mathrm{~mL})$. NADPH, EDTA, BCA kit, DTT, and Tris were of analytical grade and purchased locally. Acetonitrile and methanol (HPLC grade) were purchased from Fisher Chemicals (Fairlawn, NJ, USA).

Rat liver microsomes (RLM, Sprague-Dawley) were purchased from Wuhan Pulaite Medical and Technical Co. Ltd (M10011). Protein concentration was determined as $20 \mathrm{mg} / \mathrm{mL}$ by bicinchoninic acid (BCA) method using bovine serum albumin as standard.

\section{Instrumentation}

The LC system for LC-MS/MS was Shimadzu Nexera UPLC LC-30A containing a binary LC-30AD pump, DGU-20A5 vacuum degasser, SIL-30AC autosampler and CTO-30A thermostat column compartment. An API 4000 triple quadrupole mass spectrometer (AB SCIEX, Foster, USA) with an electrospray ionization (ESI) operated in the positive ion mode was used for analysis. Quantification was performed using multiple reaction monitoring (MRM) for the transitions $m / z$ 300. 1-209.0 for ARN and 316.2-163.0 for ARM (Fig. 2). The system was controlled by Analyst software version 1.5.1. Separation was performed on a Shimadzu XR-ODS $\mathrm{C}_{18}$ column $(2.0 \mathrm{~mm} \times 100 \mathrm{~mm}, 2.2 \mu \mathrm{m})$ with a Shimadzu ODS $\mathrm{C}_{18}$ security guard column $(5 \mathrm{~mm} \times 2.0 \mathrm{~mm}, 2.2 \mu \mathrm{m})$ maintained at $30{ }^{\circ} \mathrm{C}$ using a mobile phase containing acetonitrile and $0.1 \%$ formic acid in $10 \mathrm{mM}$ ammonium acetate $(85: 15, \mathrm{v} / \mathrm{v})$ at a flow rate of $300 \mu \mathrm{L} / \mathrm{min}$. The source temperature was maintained at $600{ }^{\circ} \mathrm{C}$ and the ESI source voltage was set at $5500 \mathrm{~V}$. Collision gas pressure was 3 units and collision energy was $17 \mathrm{~V}$.

The six enzymatic probe substrates were standardized by using Agilent 1200 (Agilent, USA) RP-HPLC system consisted of an on-line G1322A vacuum degasser, a G1311A quaternary pump, a G1329A injection valve (USA) with a sample loop of $20 \mu \mathrm{L}$, a G1314B UV-visible diode-array detector (DAD). A phenomenex $\mathrm{C}_{18}$ column (Synergi Hydro-RP 80A, $150 \mathrm{~mm} \times 4.6 \mathrm{~mm}, 4 \mu \mathrm{m}$ ) was used as stationary phase with a flow rate of $1.2 \mathrm{~mL} / \mathrm{min}$ at $30{ }^{\circ} \mathrm{C}$. The isocratic mobile phase consisted of acetonitrile and purified water containing $1 \%$ triethylamine and $0.02 \mathrm{M}$ sodium dihydrogen phosphate (40:60 for PN, CA, DM, CLZ, and MDZ; 35:65 for OMP, v/v, PH = 3.5) was respectively used for assay of $\mathrm{PN}$ (wavelength: $250 \mathrm{~nm}$ ), CLZ (282 nm), MDZ (230 nm), OMP (302 nm), CA $(278 \mathrm{~nm})$, and DM $(202 \mathrm{~nm})$.

\section{Stock solutions of chemicals}

ART and CHR were separately suspended in $0.5 \%$ carboxy methyl cellulose (CMC-Na) by sufficient emulsification to get the stock solution of $2 \mathrm{mg} / \mathrm{mL}$ strength and diluted to get the desired concentrations for each drug before it was administrated by the intramuscular injection or gavage perfusion. Master stock solutions for assay of blood concentration were individually prepared by dissolving ARN and ARM standards in acetonitrile at equivalent concentrations of $1000 \mu \mathrm{g} / \mathrm{mL}$ and were gradually diluted to $2 \mu \mathrm{g} / \mathrm{mL}$ by mobile phase for the preparation of calibration curve $(0.2-200 \mathrm{ng} / \mathrm{mL})$ and quality control (QC) samples (0.5, 10 and $160 \mathrm{ng} / \mathrm{mL}$ for ART), respectively.

For in vitro hepatic metabolic study, CHR and six enzymatic probes were separately prepared in methanol to 


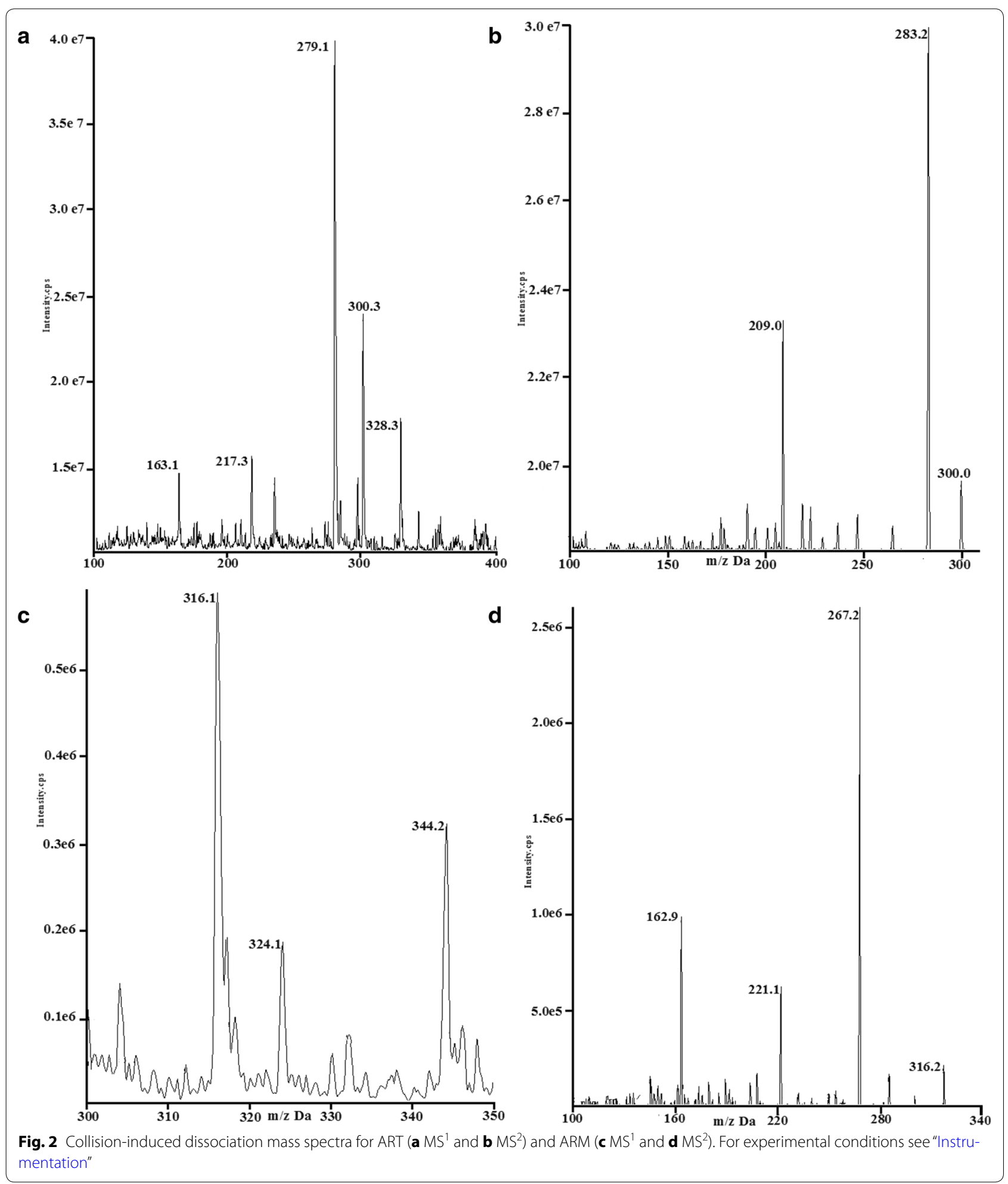

strength of $1 \mathrm{mg} / \mathrm{mL}$ stock solutions and were diluted to desired concentrations by phosphate buffers (PBS, $0.1 \mathrm{M}$, $\mathrm{PH}=7$ ) before use. QC samples were solved in inactive RLM with three concentration levels (3.0, 28.0 and 89.0 for PN; 3.4, 34.2 and 100.0 for CA; 6.0, 29.5 and 118.0 for CLZ; 3.0, 15.0 and 49.0 for MDZ; 2.7, 13.5 and 43.2 for DM). A serial of RLMs in strength of $1.25,1,0.75,0.5$, $0.25 \mathrm{mg} / \mathrm{mL}$ were gained for optimization of protein 
concentration experiment by diluting the stock protein solution in strength of $20 \mathrm{mg} / \mathrm{mL}$ (stored at $-80^{\circ} \mathrm{C}$ ). All other stock solutions were stored at $4{ }^{\circ} \mathrm{C}$ when not in use.

\section{Method validation}

Calibration curves for artemisinin were constructed by weighted least-squares linear regression $\left(1 / x^{2}\right)$ analysis of an eight-point calibration curve by plotting peak area of the analyte versus the peak area of the internal standard. The assay was validated through linearity, inter- and intra-day accuracy and precision, recovery, lower limits of quantification (LLOQ), stability, and matrix effect as usual.

Calibrations curves for six probe substrates in inactive RLM were obtained and the assay was evaluated through linearity, inter- and intra-day precision and accuracy, and recovery.

\section{Pharmacokinetics study}

Male SD rats (200-240 g) were supplied by Laboratory Animal Center of Ningxia Medical University (Grade II, Certificate No. SCXY 2011-0001). Animals were acclimatized in environmentally controlled breeding cages for at least 3 days before being used and were provided with standard laboratory food and water and were fasted for $12 \mathrm{~h}$ prior to the study. Water was freely available for rats during experiments. The experimental protocol was approved by the University Ethics Committee. All procedures involving animals were in accordance with the Regulations of the Experimental Animal Administration, State Committee of Science and Technology, People's Republic of China. 20 rats were randomly divided into 4 groups $(n=5)$. Group A was the control vehicle (ART alone) and group $\mathrm{L}, \mathrm{M}$, and $\mathrm{H}$ were the combination groups with CHR in three fixed ratios $(1: 1,1: 2$, and 1:4). The suspension of CHR was continuously administered (each time per day) to rats in group $\mathrm{L}, \mathrm{M}$, and $\mathrm{H}$ by gavage at doses of $5,10,20 \mathrm{mg} / \mathrm{kg}$ for 3 days, respectively. Group A was orally given by $0.5 \%$ CMC-Na solution once per day for 3 days. On day 3 , an intramuscular injection of ART $(5 \mathrm{mg} / \mathrm{kg})$ was subsequently administered $1 \mathrm{~h}$ after CHR or CMC-Na solution pre-exposure. Blood samples $(500 \mu \mathrm{L})$ were collected into centrifuge tube with heparin at $0,10,20,30,60,90,120,240,360,480,600$, 720 , and $1440 \mathrm{~min}$ from the orbit after the administration of ART, respectively. Two hours later, animals took food and water freely to replenish.

Plasma samples were separated by centrifugation $3500 \mathrm{~g}$ for $10 \mathrm{~min}$ and $200 \mu \mathrm{L}$ plasmas were stored at $-20{ }^{\circ} \mathrm{C}$ until analysis. To $100 \mu \mathrm{L}$ blank plasma, $50 \mu \mathrm{L}$ ARM working solution (IS, $2 \mu \mathrm{g} / \mathrm{mL}$ ), $100 \mu \mathrm{L}$ methanol aqueous solution $(50: 50, \mathrm{v} / \mathrm{v})$, and a standard solution or quality control (QC) solution were added. After vortex mixing for $1 \mathrm{~min}$, the sample was extracted with $3 \times 3 \mathrm{~mL}$ of methyl tert-butyl ether with gentle shaking for $10 \mathrm{~min}$ followed by centrifugation at $3500 \mathrm{~g}$ for $10 \mathrm{~min}$. The methyl tert-butyl ether extracts were combined and evaporated to dryness by a Pressure Gas Blowing Concentrator (multivap nitrogen evaporation system 118, Organomation Co., Ltd, USA) at $40{ }^{\circ} \mathrm{C}$. The residue was reconstituted in $100 \mu \mathrm{L}$ methanol and was filtered through a $0.22 \mu \mathrm{m}$ nylon filter. Aliquots $(10 \mu \mathrm{L})$ of the solution were injected onto the LC-MS analysis.

\section{In vivo anti-malarial efficacy}

Female Kunming mice (18-22 g) for the experiment of in vivo chemo-suppressive study were purchased from Animal Center in Academy of Military Medical Science (Grade II, Certificate No. SCXK 2002-001). Animals were divided into eight groups $(n=10)$ including placebo group (normal saline only), ART alone ( $5 \mathrm{mg} / \mathrm{kg}$ ), CHR alone $(5,10$ and $20 \mathrm{mg} / \mathrm{kg}$ ), and ART-CHR combination groups (1:1, 1:2, and 1:4). Two hours after being inoculated, they were administrated in the same delivery route and the same dosage of ART and CHR as pharmacokinetic study.

Stocks of the malaria parasite $P$. berghei $\mathrm{K} 173$ strain (chloroquine sensitive) were continuously maintained in the Microbiology Laboratory of Academy of Military Medical Sciences, China, and alternately stored by liquid nitrogen cryopreservation and blood subinoculation in mice. Approximate one million infected erythrocytes were inoculated into the enterocoelia of each mouse before use.

Twenty-four hours after the last dosing, the blood was collected from caudal vein of mice. Thin blood smear slides were air-dried, methanol-fixed, and stained in Giemsa for $40 \mathrm{~min}$. The Giemsa-stained slides were examined for counting the number of parasites in random three microscopic fields, equivalent to over 200 erythrocytes each field at $1000 \times$ magnification. Percent parasitaemia (\%) was calculated through dividing the number of total red blood cells by that of infected red blood cells. Percentage inhibition (\%) for each drug was calculated relative to placebo. Reproducibility of counts was checked by two other readers to maintain the quality control.

\section{In vitro rat liver microsome experiment}

The incubation system included $40 \mu \mathrm{L}$ of RLM (protein concentration was optimized as $1 \mathrm{mg} / \mathrm{mL}$ for $\mathrm{PN}$; $0.75 \mathrm{mg} / \mathrm{mL}$ for OPZ; $0.5 \mathrm{mg} / \mathrm{mL}$ for CA, DM, CLZ, and MDZ), $5 \mu \mathrm{L}$ of substrate solution (substrate concentration was optimized as $25 \mu \mathrm{M}$ for PN; $5 \mu \mathrm{M}$ for CA; $20 \mu \mathrm{M}$ for OPZ; $10 \mu \mathrm{M}$ for DM; $15 \mu \mathrm{M}$ for CLZ; $5 \mu \mathrm{M}$ for MDZ), and NADPH-regenerating system $(1.3 \mathrm{mM}$ 
$\mathrm{NADP}^{+}$, 3.3 mM G-6-P, 3.3 mM MgCl $2,4 \mathrm{U} / \mathrm{mL}$ G-6-PD) and a test compound (CHR, $0-50 \mu \mathrm{M})$. Total reaction volume was complemented to $200 \mu \mathrm{L}$ by PBS. After preincubating for $3 \mathrm{~min}$ at $37{ }^{\circ} \mathrm{C}$, the reaction was initiated by the addition of NADPH solution (incubation time was optimized as $45 \mathrm{~min}$ for PN; $60 \mathrm{~min}$ for CA, $15 \mathrm{~min}$ for OPZ; 20 min for DM; 90 min for CLZ; 15 min for MDZ). $200 \mu \mathrm{L}$ ice acetonitrile was added as stop reagent. After a vortex for $3 \mathrm{~min}$ and centrifugation for $10 \mathrm{~min}$ at 14,000g, an aliquot of $20 \mu \mathrm{L}$ supernatant was injected into the HPLC system at an interval of $10 \mathrm{~min}$. The amount of methanol in diluted concentrations $(<1 \%)$ had no effect on liver microsomes. All the testing groups were in three replicates $(n=3)$. Under the optimized condition, $\mathrm{V}_{\max }$, Michaelis constant $\left(K_{m}\right)$, enzyme activity $(\mathrm{E} \%)$, and $\mathrm{IC}_{50}$ values were obtained.

\section{Inhibitory type determination}

Incubation systems including $1.0 \mathrm{mg} / \mathrm{mL}$ RLM, PN (12.5, 25.0, and 50.0 $\mu \mathrm{M}), \mathrm{NPDPH}$, and $\operatorname{CHR}(0,2,4,8 \mu \mathrm{M})$ for $1 \mathrm{~A} 2$ or $0.5 \mathrm{mg} / \mathrm{mL} \operatorname{RLM}, \operatorname{MDZ}(2.5,5.0,10.0 \mu \mathrm{M})$, $\mathrm{NPDPH}$, and CHR $(0,2,4,8 \mu \mathrm{M})$ for CYP3A were employed as above $(n=3)$. A serial of Lineweaver-Burk plots by $1 /[\mathrm{S}]$ versus $1 / \mathrm{V}$ were prepared individually under different concentrations of CHR. The location of intersection point for the linear lines decided the type of inhibition. Namely, if the point lied in $\mathrm{Y}$ axis it was competitive inhibition and if it lied in $\mathrm{X}$ axis it was noncompetitive inhibition. Finally, if the linear lines were paralleled, it was uncompetitive inhibition. Concomitantly, inhibition constant $\left(K_{i}\right)$ was calculated when a second plotting was given by intercepts of the four lines versus [I] (inhibitor concentration).

\section{Data analysis}

Pharmacokinetic parameters were computed from plasma concentration-time profiles of ART by software DAS 3.0. Statistical significance of differences was analyzed by SPSS 16.0 statistical software with a significance level of $P<0.05$ and 0.01 .

\section{Results and discussion}

\section{UPLC-MS/MS method for ART}

The electrospray ionization mass spectra for ART and ARM were shown in Fig. $2 \mathrm{a}\left(\mathrm{MS}^{1}\right.$ for $\left.\mathrm{ART}\right)$, b $\left(\mathrm{MS}^{2}\right.$ for ART), c (MS ${ }^{1}$ for ARM), and d $\left(M^{2}\right.$ for ARM). Under optimized UPLC conditions, ART and ARM were eluted within 2.5 min (Fig. 3b, c). Blank plasma showed no significant interfering peaks at the retention times of each analyte (Fig. 3a). The calibration curve of ART was linear over the concentration range of $0.2-200.0 \mathrm{ng} /$ $\mathrm{mL}\left(y=0.0336 x+0.0259, r=0.9965, \mathrm{w}=1 / x^{2}\right)$ with LLOQ $0.2 \mathrm{ng} / \mathrm{mL}(\mathrm{RSD} \%=3.91, \mathrm{RE} \%=-5 \%, n=5)$.
Inter- and intra-day precision and accuracy, stability, recovery, and matrix effect data were showed in Table 1. The precision and accuracy of this method indicated that all coefficients of variation at each concentration level are below $10 \%$. The normalized matrix effects of ART at low, middle, and high concentrations were close to 1 with a low variation in accordance with international guidelines [20]. ART was found stable in plasma for at least 6 and $24 \mathrm{~h}$ at ambient temperature before and after treatment and was also stable during three freeze/thaw cycles.

\section{HPLC-UV method for six probe drugs}

Six probe drugs were well analysed within $10 \mathrm{~min}$ by HPLC-UV method without interference. Standard curves equations of six substrates in inactive RLM were displayed in Table 2. Details of method validation data were elaborately arranged in Table $3 a, b$ in different strength of QC samples.

\section{Pharmacokinetics parameters for ART in absence and presence of CHR}

Plasma concentration-time profiles of ART, in untreated rats and in pre-treated rats with different combinatory doses of CHR, were shown in Fig. 4a, b. Corresponding pharmacokinetic parameters were reported in Table 4. This study has demonstrated that the $\mathrm{AUC}_{0-\mathrm{t}}$ of $\mathrm{ART}$ in ART-CHR-L (1:1), ART-CHR-M (1:2), and ART-CHR$H(1: 4)$ groups, respectively, increased 1.44-, 1.40-, and 1.29-fold compared with ART alone $(P<0.01)$ without dose-response manner $(P>0.05$ among the combination groups). The $\mathrm{C}_{\max }$ in ART-CHR-L (1:1) and ARTCHR-M (1:2) group increased 1.64- and 1.65-fold versus control $(P<0.05)$ while no increase was found in ARTCHR-H (1:4) group. The $t_{1 / 2}$ in ART-CHR-M (1:2) group enhanced 1.68 folds compared with ART alone $(P<0.05)$. It showed that oral co-administration of CHR in combinatory ratio of 1:2 prior to intramuscular injection of ART achieved a longer $t_{1 / 2}$, a higher AUC and $C_{\max }$, and lower CLz than ART alone.

\section{Synergic anti-malarial efficacy of ART in presence of CHR}

The results showed in Table 5 suggested that parasitaemia (\%) in ART alone, ART-CHR-L, ART-CHR-M, and ART-CHR-H groups significantly decreased 1.59, 2.23, 2.53, and 2.01 folds $(P<0.05)$ compared with placebo, whereas no significance was observed when $\mathrm{CHR}$ was solely used $(P>0.05)$. CHR, therefore, has no anti-malarial effect itself. Only in ART-CHR-M group, parasitaemia (\%) and inhibition (\%), respectively, achieved 1.59-fold reduction $(P<0.05)$ and 1.63 -fold augment compared with ART alone. The result completely conformed to the pharmacokinetics study. 


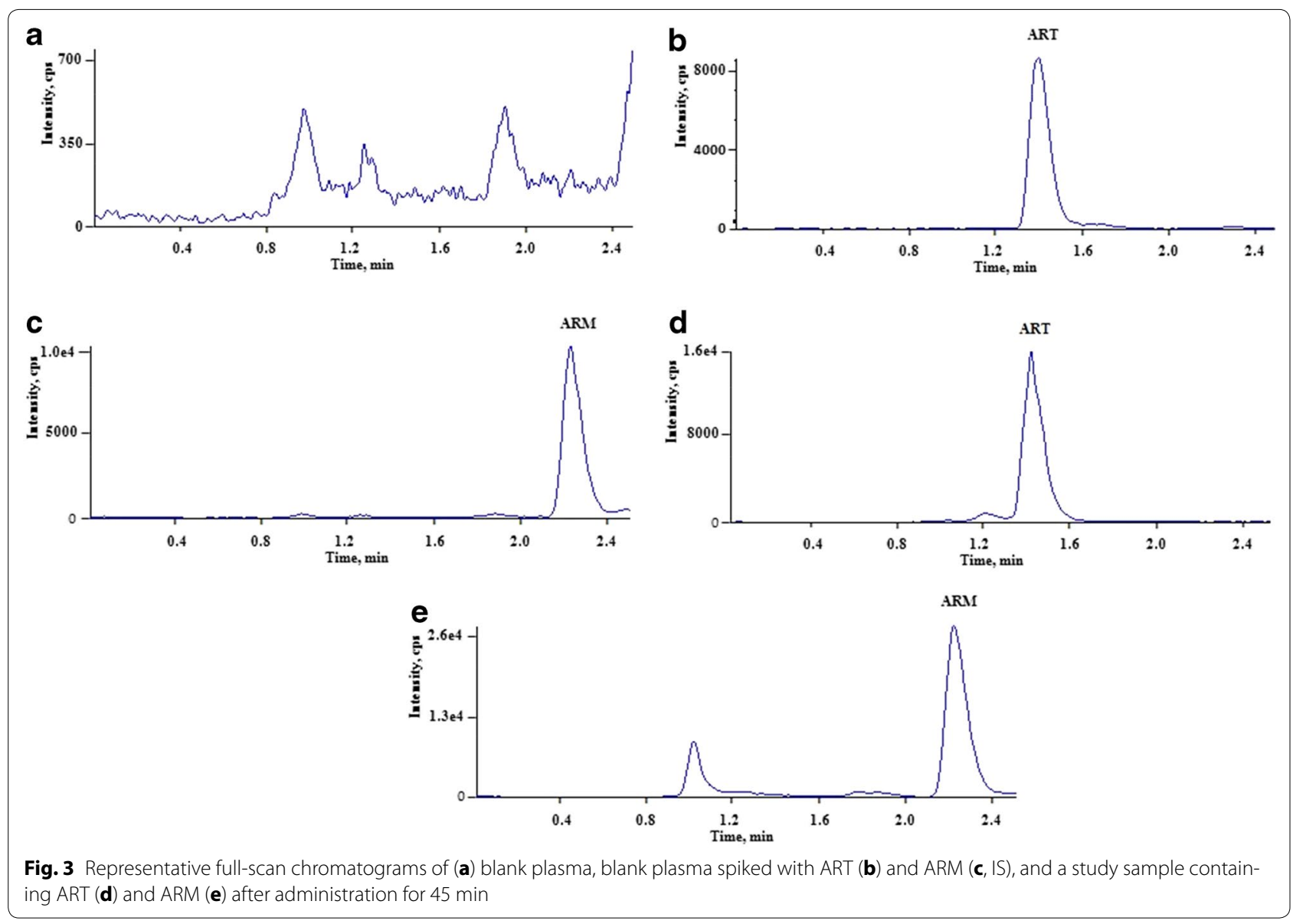

Table 1 Method validation for quantification of ART in rat plasma

\begin{tabular}{|c|c|c|c|c|c|c|c|c|}
\hline \multirow[t]{3}{*}{$\mathrm{QC}(\mathrm{ng} / \mathrm{mL})$} & \multicolumn{2}{|c|}{ Precision and accuracy (\%) } & \multicolumn{4}{|c|}{ Stability (RE\%) } & \multirow[t]{3}{*}{ Recovery (\%) } & \multirow{3}{*}{$\begin{array}{l}\text { Matrix effect }(n=5) \\
\text { Mean (\%)/RSD\% }\end{array}$} \\
\hline & \multirow{2}{*}{$\begin{array}{l}\text { Inter-day } \\
\text { RSD/RE }\end{array}$} & \multirow{2}{*}{$\begin{array}{l}\text { Intra-day } \\
\text { RSD/RE }\end{array}$} & \multicolumn{2}{|l|}{ Pre-T } & \multirow{2}{*}{$\begin{array}{l}\text { Post-T } \\
\text { RT } \\
24 \mathrm{~h}\end{array}$} & \multirow{2}{*}{$\begin{array}{l}\text { Freeze-thaw } \\
\text { for three times }\end{array}$} & & \\
\hline & & & $\begin{array}{l}\mathrm{RT} \\
6 \mathrm{~h}\end{array}$ & $\begin{array}{l}\text { RT } \\
24 \mathrm{~h}\end{array}$ & & & & \\
\hline 0.5 & $8.51 / 1.24$ & $6.57 / 1.24$ & -4.00 & -2.00 & 2.00 & 2.00 & 57.30 & $98.0 / 1.70$ \\
\hline 10 & $1.41 / 6.75$ & $6.67 /-1.90$ & 3.20 & -0.80 & -8.20 & 6.80 & 73.30 & $88.0 / 2.70$ \\
\hline 160 & $2.97 / 7.81$ & $6.99 / 0.67$ & -6.25 & -3.00 & -4.38 & 4.38 & 68.90 & $66.0 / 3.10$ \\
\hline
\end{tabular}

$R T$ room temperature, Pre-T Pre-treatment, Post-T post-treatment

Table 2 Standard curves equation of six probe substrates in inactive RLM

\begin{tabular}{llll}
\hline Probe substrates & Calibration curves & $\boldsymbol{R}$ value & Linear range $(\boldsymbol{\mu M})$ \\
\hline PN & $Y=9.486 X+0.3485$ & 0.9999 & $0.5-111$ \\
CA & $Y=6.730 X+0.7809$ & 0.9999 & $0.7-137$ \\
OPZ & $Y=4.148 X+2.1741$ & 0.9999 & $0.5-141$ \\
DM & $Y=24.868 X+5.1424$ & 0.9999 & $0.5-54$ \\
CLZ & $Y=20.918 X+1.257$ & 0.9999 & $1.0-295$ \\
MDZ & $Y=36.365 X-16.163$ & 0.9999 & $0.8-63$ \\
\hline
\end{tabular}

\section{Inhibition of CHR on CYP450 enzymes}

$K_{m}$ values for PN, CA, OPZ, DM, CLZ, and MDZ were calculated to be $30.88,8.90,24.07,28.94,19.97$, and $7.88 \mu \mathrm{M}$ with $\mathrm{V}_{\max } 0.14,0.12,0.58,0.20,0.18,0.81 \mathrm{nmol} /$ $\mathrm{min} / \mathrm{mg}$ proteins, respectively. The working substrate concentrations for PN, CA, OPZ, DM, CLZ, and MDZ, therefore, were confirmed to be $25,5,20,10,15$, and $5 \mu \mathrm{mol} \mathrm{L}^{-1}$ (lower than $K_{m}$ ).

Graphs showed in Fig. 5 and data in Table 6 demonstrated that CHR has a significant inhibition $(P<0.01)$ on CYP1A2 and CYP2C19 in a concentration-dependence 


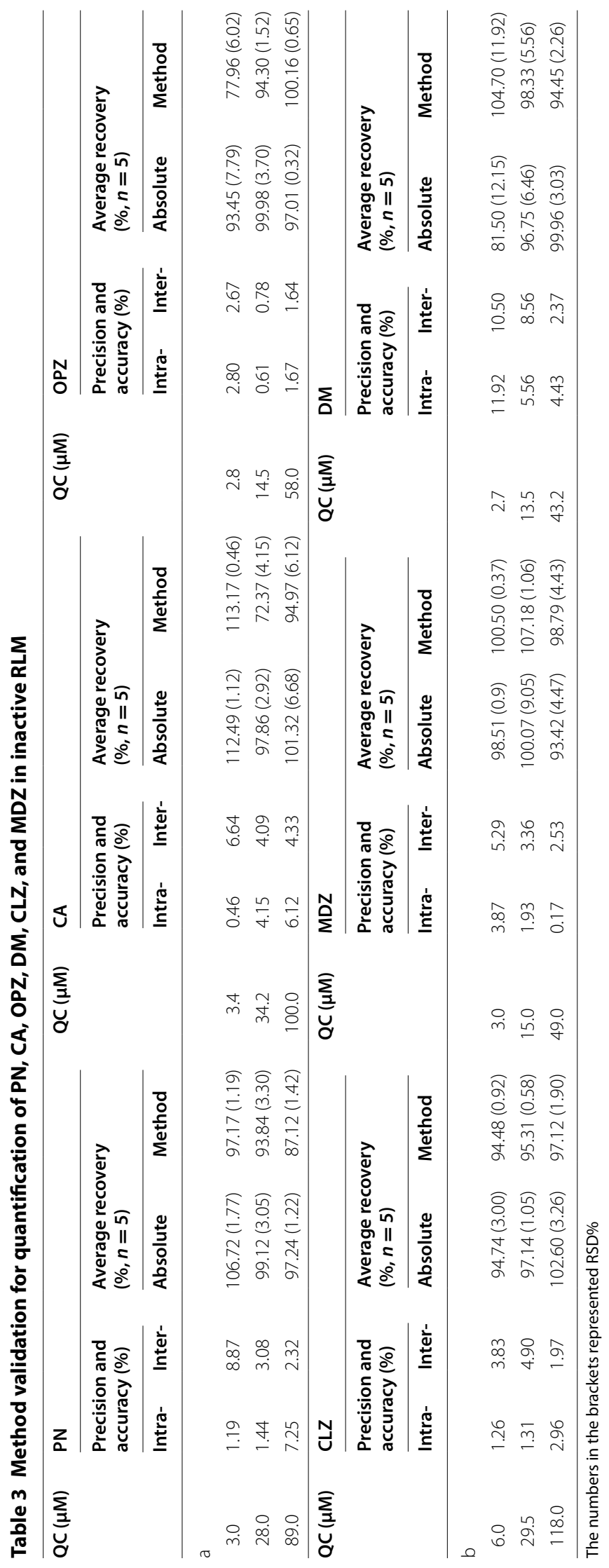



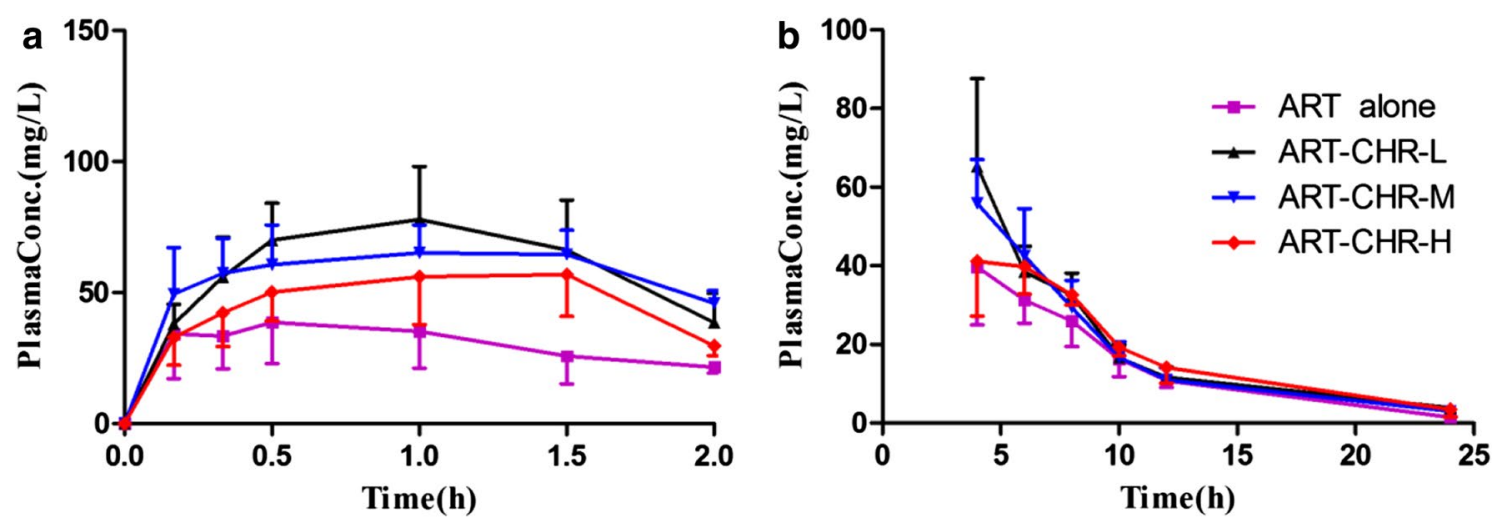

Fig. 4 Mean ( $\pm S D$ ) plasma concentration-time profiles of ART ( $n=5)$ in ART alone (square points), ART-CHR-L (triangular points), ART-CHR-M (inverted triangular points), and ART-CHR-H (diamond points). a before $2 \mathrm{~h}, \mathbf{b}$ after $2 \mathrm{~h}$

Table 4 Plasma concentration-time profiles of ART with or without $\mathrm{CHR}(n=5)$

\begin{tabular}{|c|c|c|c|c|}
\hline Ratio & $t_{1 / 2}(\mathrm{~h})$ & $A U C_{0-t}[\mathrm{mg} /(\mathrm{L} \mathrm{h})]$ & $C_{\max }(m g / L)$ & CLz (L/h/kg) \\
\hline ART alone & $3.62 \pm 0.86$ & $390.58 \pm 32.46$ & $51.58 \pm 9.47$ & $0.013 \pm 0.001$ \\
\hline ART-CHR-L (1:1) & $3.89 \pm 1.93$ & $562.20 \pm 101.50^{* *}$ & $84.76 \pm 19.52^{*}$ & $0.009 \pm 0.002^{*}$ \\
\hline ART-CHR-M (1:2) & $6.07 \pm 2.04^{* \#}$ & $547.68 \pm 76.59^{* *}$ & $85.22 \pm 29.56^{*}$ & $0.009 \pm 0.001^{* *}$ \\
\hline ART-CHR-H (1:4) & $3.28 \pm 0.77$ & $505.21 \pm 54.88^{* *}$ & $63.16 \pm 16.50$ & $0.010 \pm 0.001^{* *}$ \\
\hline
\end{tabular}

Data are mean values $\pm S D$ in five rats

Significantly different from ART alone: ${ }^{*} P<0.05,{ }^{* *} P<0.01$

Significantly difference from ART-CHR-H: ${ }^{\#} P<0.05$

Table 5 In vivo antimalaria pharmacodynamic effect of ART in combination with CHR

\begin{tabular}{|c|c|c|c|c|c|}
\hline \multirow[t]{2}{*}{ Groups } & \multirow[t]{2}{*}{ Dosage $(\mathrm{mg} / \mathrm{kg})$} & \multirow[t]{2}{*}{ Parasitemia $(\%, n=10)$} & \multicolumn{2}{|l|}{$P$ value } & \multirow[t]{2}{*}{ Inhibition $(\%, n=10)$} \\
\hline & & & placebo & ART alone & \\
\hline Placebo & 0 & $22.15 \pm 6.25$ & - & - & \\
\hline CHR-L & 5 & $19.74 \pm 13.33$ & 0.62 & - & - \\
\hline CHR-M & 10 & $17.19 \pm 5.45$ & 0.05 & - & - \\
\hline $\mathrm{CHR}-\mathrm{H}$ & 20 & $18.32 \pm 8.86$ & 0.25 & - & - \\
\hline ART alone & 5 & $13.95 \pm 7.89^{*}$ & 0.01 & - & 37.06 \\
\hline ART-CHR-L & $5(1: 1)$ & $9.95 \pm 5.36^{*}$ & $2.73 \times 10^{-5}$ & 0.07 & 55.10 \\
\hline ART-CHR-M & $10(1: 2)$ & $8.75 \pm 4.25^{*, \#}$ & $2.16 \times 10^{-6}$ & 0.04 & $60.51^{\#}$ \\
\hline ART-CHR-H & $20(1: 4)$ & $11.00 \pm 4.98^{*}$ & $2.72 \times 10^{-5}$ & 0.18 & 50.36 \\
\hline
\end{tabular}

* $P$ values of drugs in relation to placebo: $P<0.05$

$\# P$ values of ART-CHR combination groups in relation to ART alone: $P<0.05$

manner and enzyme activity (\%) decreased from $(21.64 \pm 1.05) \%$ to $(4.05 \pm 1.41) \%$ for CYP1A2, and from $(41.98 \pm 0.35) \%$ to $(0.60 \pm 0.11) \%$ for CYP2C19 when $\mathrm{CHR}$ concentration increased from 0 to $50 \mu \mathrm{M}$. As for CYP2E1, CHR had a significant inhibition in a doseresponse manner only after CHR concentration exceeded
$4 \mu \mathrm{M}(P<0.05)$. Most interestingly, $0.5 \mu \mathrm{M}$ of CHR significantly increased the enzyme activity (\%) of CYP3A from $48.27 \pm 0.93(0.0 \mu \mathrm{M})$ to $59.44 \pm 2.55(P<0.01)$, and it slowly decreased to $57.15 \pm 0.93(P<0.01), 52.02 \pm 3.07$ $(P>0.05)$, and $51.37 \pm 0.84(P>0.05)$ after CHR concentration added from 1.0 to $2.0 \mu \mathrm{M}$. An inhibition of 


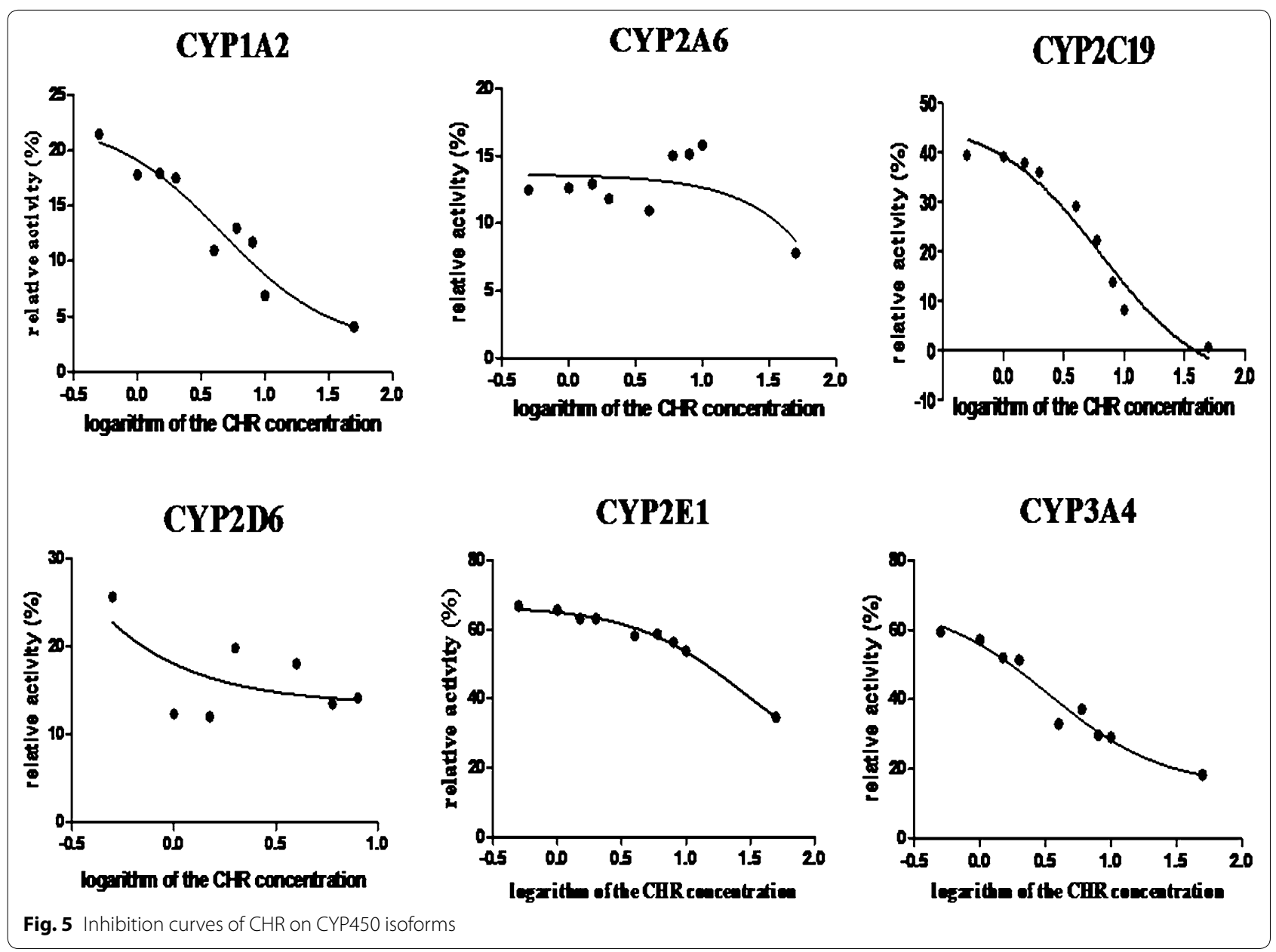

Table 6 Effects of CHR on cytochrome P450 isoforms in rat liver microsomes in vitro

\begin{tabular}{|c|c|c|c|c|c|c|}
\hline \multirow[t]{2}{*}{$\mathrm{CHR} / \mu \mathrm{M}$} & \multicolumn{6}{|c|}{ Enzyme activity (\%) } \\
\hline & $1 \mathrm{~A} 2$ & $2 A$ & $2 C 19$ & 2D6 & $2 \mathrm{E} 1$ & $3 A$ \\
\hline 0 & $21.64 \pm 1.05$ & $14.45 \pm 8.11$ & $41.98 \pm 0.35$ & $19.25 \pm 11.87$ & $69.82 \pm 7.31$ & $48.27 \pm 4.71$ \\
\hline 0.5 & $21.46 \pm 2.83^{*}$ & $12.47 \pm 1.91$ & $39.56 \pm 1.35^{* *}$ & $25.62 \pm 3.56$ & $66.93 \pm 1.82$ & $59.44 \pm 2.55^{* *}$ \\
\hline 1.0 & $17.80 \pm 3.07^{* *}$ & $12.62 \pm 1.35$ & $38.92 \pm 0.56^{* *}$ & $12.34 \pm 12.08$ & $65.76 \pm 1.72$ & $57.15 \pm 0.93^{* *}$ \\
\hline 1.5 & $17.93 \pm 1.15^{* *}$ & $12.92 \pm 0.86$ & $37.64 \pm 2.31^{* *}$ & $12.03 \pm 6.33$ & $63.23 \pm 2.40$ & $52.02 \pm 3.07$ \\
\hline 2 & $17.50 \pm 0.58^{* *}$ & $11.82 \pm 1.76$ & $36.14 \pm 2.19^{* *}$ & $19.81 \pm 0.64$ & $63.22 \pm 2.06$ & $51.37 \pm 0.84$ \\
\hline 4 & $10.96 \pm 3.56^{* *}$ & $10.94 \pm 6.85$ & $29.22 \pm 1.06^{* *}$ & $18.03 \pm 4.02$ & $58.21 \pm 5.63^{*}$ & $32.94 \pm 2.87^{* *}$ \\
\hline 6 & $12.99 \pm 1.56^{* *}$ & $15.03 \pm 0.33$ & $21.40 \pm 3.06^{* *}$ & $13.49 \pm 10.99$ & $58.79 \pm 2.29^{*}$ & $37.18 \pm 0.46^{* *}$ \\
\hline 8 & $11.72 \pm 2.81^{* *}$ & $15.12 \pm 0.96$ & $13.84 \pm 0.49^{* *}$ & $14.16 \pm 7.44$ & $56.44 \pm 5.63^{*}$ & $29.70 \pm 4.12^{* *}$ \\
\hline 10 & $6.89 \pm 1.56^{* *}$ & $15.82 \pm 1.62$ & $8.30 \pm 1.04^{* *}$ & ND & $53.81 \pm 2.02^{*}$ & $29.08 \pm 3.76^{* *}$ \\
\hline 50 & $4.05 \pm 1.41^{* *}$ & $7.79 \pm 0.95$ & $0.60 \pm 0.11^{* *}$ & ND & $34.62 \pm 6.25^{*}$ & $18.27 \pm 14.55^{* *}$ \\
\hline
\end{tabular}

$N D$ no detection

Compared with control group (no CHR)

${ }^{*} P<0.05$, ${ }^{*} P<0.01$ 


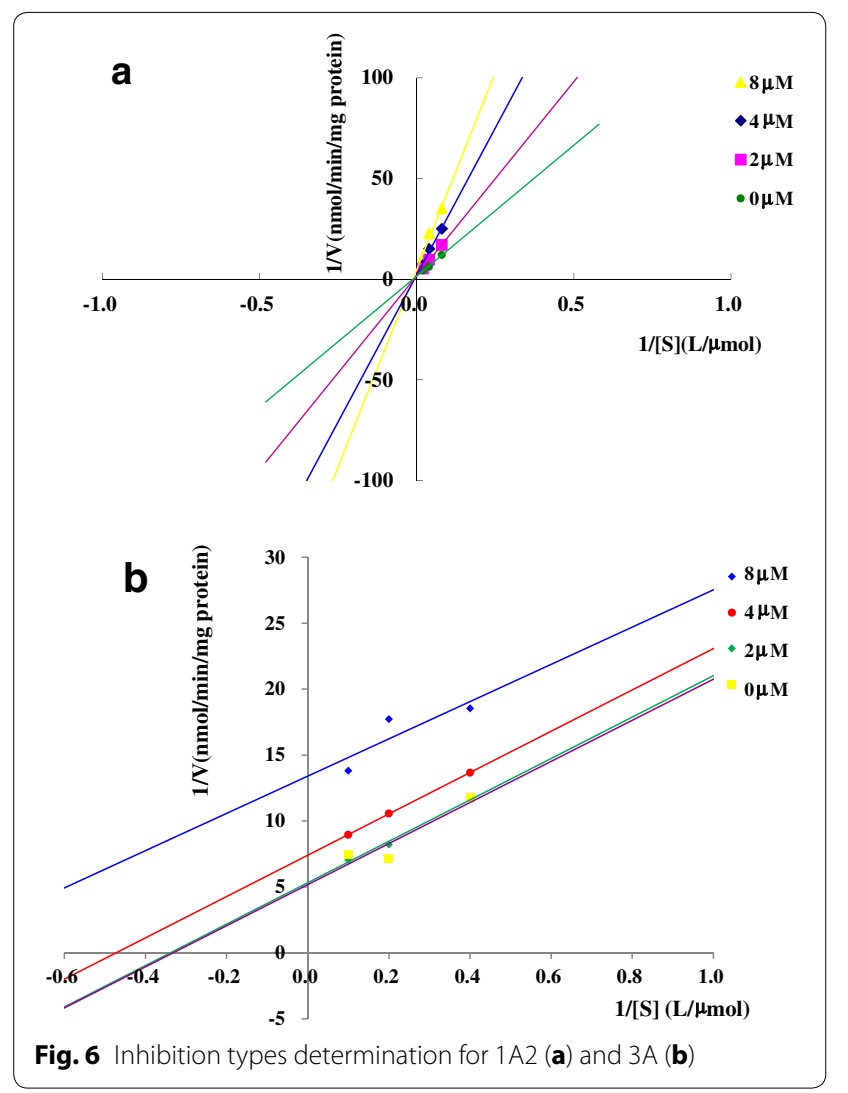

CHR on CYP3A subsequently $(P<0.01)$ emerged after the concentration exceeded $4 \mu \mathrm{M}$ as well as CYP2E1. No impact of CHR on CYP2A6 and CYP2D6 were found in this paper $(P>0.05)$.

$\mathrm{IC}_{50}$ values were computed to $4.61 \mu \mathrm{M}$ for $1 \mathrm{~A} 2$, $6.23 \mu \mathrm{M}$ for $2 \mathrm{C} 19,3.38 \mu \mathrm{M}$ for $3 \mathrm{~A}$, and $28.17 \mu \mathrm{M}$ for 2E1 by GraphPad Prism 5.0 software. CHR is a moderate inhibitor on 1A2, 3A, and $2 \mathrm{C} 19\left(1<\mathrm{IC}_{50}<10 \mu \mathrm{M}\right)$ and a weak inhibitor on $2 \mathrm{E} 1\left(\mathrm{IC}_{50}>10 \mu \mathrm{M}\right)$ according to the research [21].

\section{Inhibitory type determination for $1 \mathrm{~A} 2$ and $3 \mathrm{~A}$}

Figure $6 \mathrm{a}, \mathrm{b}$ showed that the type of $\mathrm{CHR}$ as an inhibitor on CYP1A2 belonged to non-competitive (intersection point lied in $\mathrm{X}$ axis) and that on CYP3A was uncompetitive inhibition (parallel lines). $K_{i}$ was determined to $4.07 \mu \mathrm{M}$ for CYP1A2 $(y=33.221 x+135.2, r=0.9904)$ and $3.71 \mu \mathrm{M}$ for CYP3A $(y=0.5416 x+2.012$, $r=0.9600)$.

Inhibition of inhibitors on enzymes has two types, namely irreversible and reversible inhibition. The latter includes competitive, noncompetitive, uncompetitive and mixed competitive inhibition (showed in Fig. 7). The dynamic characteristic of uncompetitive inhibition (substrate is indispensable for the inhibition) is that both $\mathrm{V}_{\max }$ and $K_{m}$ decreased when inhibitor concentration

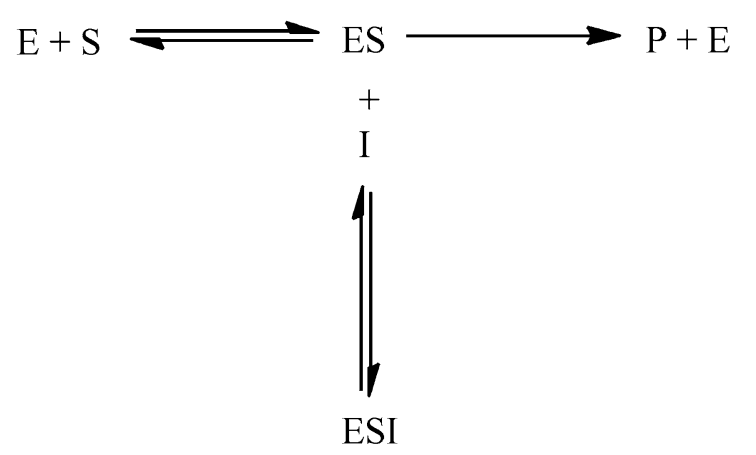

Uncompetitive inhibition

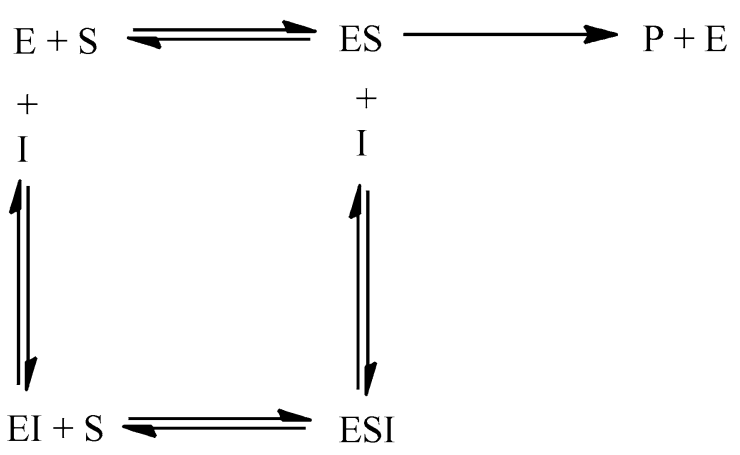

Noncompetitive inhibition

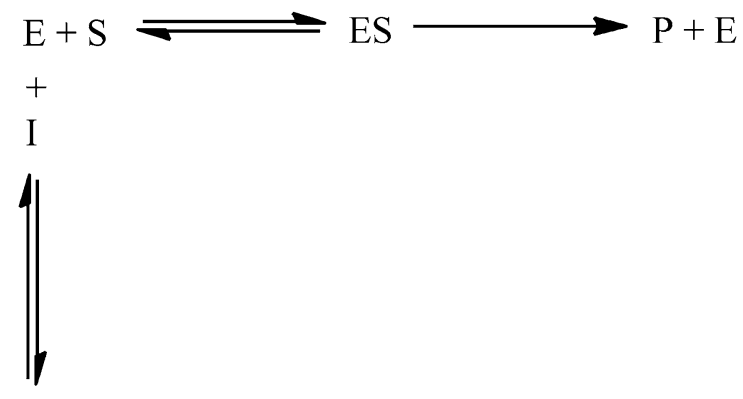

EI

Competitive inhibition

Fig. 7 Diagrammatic sketches showed that the difference among uncompetitive, noncompetitive inhibition, and competitive inhibition 
increased. This type of inhibition is infrequency in single substrate catalysis of enzyme. This study demonstrated that $\mathrm{CHR}$ acted as an on uncompetitive inhibitor of CYP3A activity and as a non-competitive inhibitor of CYP1A activity. The results are in accordance with the literatures [22, 23]. Especially, the fact that CHR inhibited CYP3A in rat liver microsome in an uncompetitive manner might be meaningful for us to partly explain why CHR has a partial dose-dependent manner in pharmacokinetics and anti-malarial efficacy studies. More interestingly, initially increased enzyme activity of CYP3A under a low concentration of CHR treatment was observed in the inhibition experiment using RLM. The authors speculate that the phenomenon might be closely related to the uncompetitive inhibition type of CHR on CYP3A. CHR exclusively bonded to enzyme-substrate complex (ES) instead of enzyme itself; therefore, when inhibitor is added into the reaction, the equilibrium reaction will shift to the product generation direction, which enhances the production of ES complex instead. When CHR concentration is very low, ES complex may not be fully bonded. The surplus will continue to be decomposed to enzyme and product by enzymolysis. So the enzyme activity initially increased a little compared with the control. As CHR concentrations increased, however, the activity slowly decreased and then inhibition effect was finally observed due to the binding between CHR and ES complex and therefore the hindering of the enzymolysis. The increased activity might, therefore, not be activated by induction of CHR on CYP3A but possibly depends on the shifting of equilibrium reaction. Further research should be carried out to demonstrate the hypothesis.

\footnotetext{
Abbreviations

UPLC: ultra-high performance liquid chromatography; MS: mass spectrometry; MS/MS: tandem mass spectrometry; CHR: chrysosplenetin; ART: artemisinin; ARM: artemether; ACT: artemisinin-based combination therapy; NADPH: nicotinamide adenine dinucleotide phosphate; BCA: bicinchoninic acid; DTT: dithiothreitol; Tris: tris(hydroxymethyl)metyl aminomethane.

\section{Authors' contributions}

SW contributed to the design of the research, the performance of the ART assay in rat plasma by UPLC-MS/MS and the determination of probe substrates in liver microsomes, the analysis of the data. HJ executed the determination of probe substrates in liver microsomes and inhibition type assessment. BY participated in the data analysis. LM contributed to the critical revision of the manuscript. ZB performed the activities of ART alone and ART combination with $\mathrm{CRH}$ in vivo. $\mathrm{XL}$ contributed to the pharmacokinetics of ART when combined with CHR or without CHR. HD advised in the design of the study and adjusted the manuscript. XY helped to draft the manuscript. CL participated in the data analysis. XW designed the protocol, analysis the data and helped in the draft of the manuscript. JC conceived of the study, coordinated its execution, analysed the data, and wrote the paper. All authors read and approved the final manuscript.
}

\section{Author details}

${ }^{1}$ School of Pharmacy, Ningxia Medical University, 1160\# Shengli Street, Xingqing District, Yinchuan 750004, People's Republic of China. ${ }^{2}$ Institute of Clinical Pharmacology, General Hospital of Ningxia Medical University,
Yinchuan, People's Republic of China. ${ }^{3}$ Institute of Epidemic Disease, Academy of Military Medical Sciences, Beijing, People's Republic of China.

\section{Acknowledgements}

This work was supported by the National Science Foundation of China (No. 30960465 and No. 81560580).

\section{Competing interests}

The authors declare that they have no competing interests.

Received: 17 December 2014 Accepted: 6 October 2015

Published online: 04 November 2015

\section{References}

1. Meshnick SR, Taylor TE, Kamchonwongpaisan S. Artemisinin and the antimalarial endoperoxidases: from herbal remedy to targeted chemotherapy. Microbiol Rev. 1996;60:301-15.

2. World Health Organization: Guidelines for the Treatment of Malaria. WHO/HTM/MAL/2006, 1108.

3. Gbotosho GO, Okuboyejo TM, Happi CT, Sowunmi A. Recrudescent Plasmodium falciparum infections in children in an endemic area following artemisinin-based combination treatments: implications for disease control. Asian Pac J Trop Dis. 2011;1:195-202.

4. Asimus S, Elsherbiny D, Hai TN, Jansson B, Huong NV, Petzold MG, et al. Artemisinin antimalarials moderately affect cytochrome P450 enzyme activity in healthy subjects. Fundam Clin Pharmacol. 2007;21:307-16.

5. Asimus S, Hai TN, Huong NV, Ashton M. Artemisinin and CYP2A6 activity in healthy subjects. Eur J Clin Pharmacol. 2008;64:283-92.

6. Ashton M, Hai TN, Sy ND, Huong DX, Huong NV, Nieu NT, et al. Artemisinin pharmacokinetics is time-dependent during repeated oral administration in healthy male adults. Drug Metab Dispos. 1998;26:25-7.

7. Zang Meitong, Zhu Fanping, Li Xinxiu, Yang Aijuan, Xing Jie. Auto-induction of phase phase I and phase II metabolism of artemisinin in healthy Chinese subjects after oral administration of a new artemisinin-piperaquine fixed combination. Malar J. 2014;13:214.

8. Svensson US, Ashton M. Identification of the human cytochrome P450 enzymes involved in the vitro metabolism of artemisinin. Br J Clin Pharmacol. 1999;48:528-35.

9. El-Lakkany NM, Seifel-Din SH, Badawy AA, Ebeid FA. Effect of artemether alone and in combination with grapefruit juice on hepatic drug-metabolising enzymes and biochemical aspects in experimental Schistosoma mansoni. Int J Parasitol. 2004;34:1405-12.

10. Elford BC, Roberts MF, Phillipson JD, Wilson RJ. Potentiation of the antimalarial activity of qinghaosu by methoxylated flavones. Trans R Soc Trop Med Hyg. 1987;81:434-6.

11. Bhakuni RS, Jain DC, Sharma RP, Kumar S. Secondary metabolites of Artemisia annua and their biological activity. Curr Sci. 2001;80:35-48.

12. Quintieri L, Palatini P, Nassi A, Ruzza P, Floreani M. Flavonoids diosmetin and luteolin inhibit midazolam metabolism by human liver microsomes and recombinant CYP3A4 and CYP3A5 enzymes. Biochem Pharmacol. 2008;75:1426-37.

13. Fan $\mathrm{H}$, Zhong $\mathrm{GP}$, Zhao LZ, Bi HC, Huang M. Flavonoids inhibit six cytochrome P450 enzymes from human liver microsomes as analyzed by LC-MS/MS method. Chin J New Drugs. 2009;18:2340-8.

14. Rodriguez E, Carman NJ, Velde GV, McReynolds JH, Mabry TJ, Irwin MA, et al. Methoxylated flavonoids from Artemisia. Phytochemistry. 1972;11:3509-14

15. Djermanović M, Jokić A, Mladenović S, Stefanović M. Quercetagetin 6, 7, 3', 4'-tetramethyl ether: a new flavonol from Artemisia annua. Phytochemistry. 1873;1975:14.

16. Marco JA, Barbera O, Rooriguez S, Domingo C, Adell J. Flavoids and other phenolics from Artemisia hispanica. Phytochemistry. 1988;27:3155-9.

17. Horie T, Kawamura Y, Yamada T. Revised structure of a natural flavone from Artemisia lanata. Phytochemistry. 1989;28:2869-71.

18. Ahmad VU, Khan MA, Baqai FT, Tareen RB. Santoflavone, A 5-deoxyflavonoid from Achillea santolina. Phytochemistry. 1995;38:1305-7.

19. Chen J, Zhou YB, Zhang X, Huang L, Sun W, Wang JH. Chemical constituents of Artemisia annua L. J Shengyang Pharm Univ. 2008;25:866-70. 
20. Viswanathan CT, Bansal S, Booth B, DeStefano AJ, Rose MJ, Saistad J, et al. Quantitative bioanalytical methods validation and implementation: best practices for chromatographic and ligand binding assays. Pharm Res. 2007:24:1962-73.

21. White R. High-throughput screening in drug metabolism and pharmacokinetic support of drug discovery. Annu Rev Pharmacol Toxicol. 2000;40:133-57
22. Frank LH, Grosvick BE, Goksøyr A, Celander MC. Interactions between xenoestrogens and ketoconazole on hepatic CYP1A2 and CYP3A, in juvenile Atlantic cod (Gadus morhua). Comp Hepatol. 2005;4:2.

23. Obermeier MT, White RE, Yang CS. Effects of bioflavonoids on hepatic P450 activities. Xenobiotica. 1995;25:575-84.

Submit your next manuscript to BioMed Central and take full advantage of:

- Convenient online submission

- Thorough peer review

- No space constraints or color figure charges

- Immediate publication on acceptance

- Inclusion in PubMed, CAS, Scopus and Google Scholar

- Research which is freely available for redistribution

Submit your manuscript at

www.biomedcentral.com/submit

() Biomed Central 